\title{
LOWER BOUNDS FOR THE MODULI OF PATH FAMILIES WITH APPLICATIONS TO NON-TANGENTIAL LIMITS OF QUASICONFORMAL MAPPINGS
}

\author{
MATTI VUORINEN
}

\section{Introduction}

Given a set $E \subset R^{n}$ and a point $x \in R^{n}, n \geqq 2$, we denote by cap dens $(E, x)$ and cap $\overline{\operatorname{dens}}(E, x)$ the lower and upper $n$-capacity densities of $E$ at $x$. These concepts will be defined in Section 2 by means of $n$-moduli of path families, and therefore one could as well regard these as "lower and upper $n$-modulus densities". For an alternative definition involving $n$-capacities of condensers, we refer the reader to Martio and Sarvas [7] and to Remark 2.6.

Let now $E_{1}$ and $E_{2}$ be two sets in $R^{n}$ with cap dens $\left(E_{j}, 0\right)=\delta_{j}>0, j=1,2$, and for $r>0$ let $\Gamma_{r}$ denote the path family whose elements join $E_{1}$ and $E_{2}$ in $R^{n} \backslash \bar{B}^{n}(r)$ in the sense of Section 2, and let $M\left(\Gamma_{r}\right)$ denote the $n$-modulus of $\Gamma_{r}$. Our main result is the following lower bound for $M\left(\Gamma_{r}\right)$ : there exists a constant $c>0$ depending only on $\delta_{1}, \delta_{2}$, and $n$ such that for small $r>0$

$$
M\left(\Gamma_{r}\right) \geqq c \log \frac{1}{r} .
$$

This lower bound is well known only in some particular cases, e.g. when $E_{1}$ and $E_{2}$ are connected sets joining 0 and the boundary of the unit ball $B^{n}$. The estimate (1.1), together with other lower bounds of Section 3, is proved by means of the so-called comparison principle for the modulus. The comparison principle was introduced by Näkki in [8] and it is closely related to a lemma of Martio, Rickman, and Väisälä [6, 3.11].

In Section 4 we shall use the method of Section 3 to study the following problem. Let $f$ be a quasiconformal mapping of $B^{n}$, let $b \in \partial B^{n}$, let $E_{j} \subset B^{n}$ be a set with $b \in \bar{E}_{j}, j=1,2$, and assume that $f(x)$ tends to a limit $\alpha_{j}$ as $x$ approaches $b$ through $E_{j}, j=1,2$. How thick must the sets $E_{j}$ be at $b$ in order that $\alpha_{1}=\alpha_{2}$ ? It is easy to see that this is the case if $E_{1}$ and $E_{2}$ are non-degenerate connected sets. We shall show that even the considerably weaker conditions cap dens $\left(E_{1}, b\right)>0$ and cap $\overline{\operatorname{dens}}\left(E_{2}, b\right)>0$ imply $\alpha_{1}=\alpha_{2}$. As regards the sharpness of these conditions, 
we shall show that the former condition cannot be replaced by the weaker condition cap $\overline{\operatorname{dens}}\left(E_{1}, b\right)>0$. Problems of this kind are related to the results of [13], and the main result of Section 4, Theorem 4.12, gives us a new proof for a quasiconformal version of J. L. Doob's theorem [1, Theorem 4] (cf. also [13, Section 5]).

The results of this paper were announced in [14], where also an application of (1.1) to quasiregular mappings was given.

\section{Preliminary results}

2.1. Notation. Throughout the paper we assume that $n$ is a fixed integer and $n \geqq 2$. We denote the $n$-dimensional euclidean space by $R^{n}$ and its one-point compactification by $\bar{R}^{n}=R^{n} \cup\{\infty\}$. All topological operations are performed with respect to $\bar{R}^{n}$ unless otherwise mentioned. Balls and spheres centered at $x \in R^{n}$ and with radius $r>0$ are denoted, respectively, by

$$
\begin{aligned}
B^{n}(x, r) & =\left\{z \in R^{n}:|z-x|<r\right\}, \\
S^{n-1}(x, r) & =\left\{z \in R^{n}:|z-x|=r\right\} .
\end{aligned}
$$

We employ the abbreviations $B^{n}(r)=B^{n}(0, r), B^{n}=B^{n}(1), S^{n-1}(r)=S^{n-1}(0, r)$, and $S^{n-1}=S^{n-1}(1)$. For $r>s>0$ we denote the spherical ring $B^{n}(r) \backslash \bar{B}^{n}(s)$ by $R(r, s)$.

2.2. Path families and their modulus. A path is a continuous nonconstant mapping $\gamma:[0,1] \rightarrow A$, where $A$ is a subset of $\bar{R}^{n}$. The point set $\gamma[0,1]$ will be denoted by $|\gamma|$. Given sets $E, F$, and $G$ in $\bar{R}^{n}$, we let $\Delta(E, F ; G)$ denote the family of all paths $\gamma$ joining $E$ and $F$ in $G$ in the following sense: $\gamma(0) \in E, \gamma(1) \in F$ and $|\gamma| \subset G$. For the definition and basic properties of the (n-)modulus $M(\Gamma)$ of a path family $\Gamma$ we refer the reader to Väisälä's book [10, Chapter 1]. Given a set $E \subseteq R^{n}, r>0$, and $x \in R^{n}$, we introduce the abbreviation

$$
M(E, r, x)=M\left(\Delta\left(S^{n-1}(x, 2 r), \bar{B}^{n}(x, r) \cap E ; R^{n}\right)\right) .
$$

Let $u \in R^{n}$ and $0<a<b$ and let $\Gamma$ be a path family such that $\mid \overline{\gamma \mid} \cap S^{n-1}(u, a) \neq$ $\emptyset \neq \overline{\gamma \mid} \cap S^{n-1}(u, b)$ for every $\gamma \in \Gamma$. Then the upper bound

$$
M(\Gamma) \leqq \omega_{n-1}\left(\log \frac{b}{a}\right)^{1-n}
$$

holds $[10,6.4,7.5]$ and here $\omega_{n-1}$ is the surface area of $S^{n-1}$.

If $E \subset R^{n}$ and $x \in R^{n}$, we define the lower and upper (n-)capacity densities of $E$ at $x$ by

$$
\begin{aligned}
& \text { cap dens }(E, x)=\liminf _{r \rightarrow 0} M(E, r, x), \\
& \text { cap } \overline{\operatorname{dens}}(E, x)=\limsup _{r \rightarrow 0} M(E, r, x) .
\end{aligned}
$$


2.6. Remark. Martio and Sarvas considered in [7] the condition cap $\overline{\text { dens }}(E, x)=0$ for compact $E$. The definition in [7] was based on the use of condensers and their $n$-capacities. It follows from Ziemer [15] that the definition of Martio and Sarvas is, for compact $E$, equivalent to (2.5).

The most important lower bounds for the moduli of path families are given by the following lemma. This result is often called the (spherical) cap-inequality and was proved by Gehring (cf. [10, Chapter 10]).

2.7. Lemma. Let $E$ and $F$ be disjoint non-empty subsets of the sphere $S=S^{n-1}(x, r)$ and let $M^{S}$ be the n-modulus on $S$. Then

$$
M^{s}(\Delta(E, F ; S)) \geqq c_{n} / r,
$$

where $c_{n}$ is a positive constant, as in [10, (10.11)], depending only on $n$.

Throughout the entire paper we let $c_{n}$ denote this constant. The cap-inequality yields the following standard lower bounds for the quantities $M(E, r, 0)$, which will be frequently used in the sequel.

The euclidean diameter of $A \subset R^{n}$ is denoted by $d(A)$.

2.8. Lemma. Let $E$ be a set in $R^{n}$ and let $r>0$. Suppose that there is a connected set $E_{r} \subset \bar{B}^{n}(r) \cap E$. Then

$$
M(E, r, 0) \geqq c_{n} \log \frac{4 r+d\left(E_{r}\right)}{4 r-d\left(E_{r}\right)} .
$$

If $\bar{E}_{r} \cap S^{n-1}(r) \neq \emptyset$ and $\bar{E}_{r} \cap S^{n-1}(s) \neq \emptyset$ for some $s \in(0, r)$, then

$$
M(E, r, 0) \geqq c_{n} \log \frac{2 r-s}{r} .
$$

Proof. The lemma was proved in [13]. For completeness we will prove (2). To prove the second inequality fix $u \in \bar{E}_{r} \cap S^{n-1}(s)$ and $v \in \bar{E}_{r} \cap S^{n-1}(r)$ and choose a line $L$ through $u$ and $v$. Let $w \in L \cap S^{n-1}(2 r)$ be such that $|v-w| \leqq|u-w|$. Let $p$ and $q$ denote the lengths of the projections of $u-v$ and $v-w$ on the line through 0 and $v$. We get by the cap-inequality (cf. [10, 10.12])

$$
M(E, r, 0) \geqq c_{n} \log \frac{|u-v|+|v-w|}{|v-w|} \geqq c_{n} \log \left(\frac{p}{q}+1\right) \geqq c_{n} \log \frac{2 r-s}{r},
$$

where we have applied the obvious estimate $p / q \geqq(r-s) / r$.

Lemma 2.8 gives us an example of a situation where one obtains a lower bound for the modulus of a path family joining two sets by means of the cap-inequality. In many cases this is not possible; see e.g. the situation described at the beginning of Section 3. In such cases we shall apply the next lemma, which, following Näkki [8, 3.3], we shall call the comparison principle for the modulus. Martio, Rickman, and Väisälä have used the idea behind Lemma 2.9 in the proof of Lemma 3.11 in [6]. 
2.9. Lemma. Let $F_{1}, F_{2}$, and $F_{3}$ be three sets in $\bar{R}^{n}$ and write $\Gamma_{i j}=\Delta\left(F_{i}, F_{j} ; R^{n}\right)$, $1 \leqq i, j \leqq 3$. If there exist $x \in R^{n}$ and $0<a<b$ such that $F_{1}, F_{2} \subset \bar{B}^{n}(x, a)$ and $F_{3} \subset R^{n} \backslash B^{n}(x, b)$, the following estimate holds:

$$
M\left(\Gamma_{12}\right) \geqq 3^{-n} \min \left\{M\left(\Gamma_{13}\right), M\left(\Gamma_{23}\right), c_{n} \log \frac{b}{a}\right\} .
$$

\section{Lower bounds for the moduli of path families}

Let $E_{1}$ and $E_{2}$ be two sets in $R^{n}$ with $M\left(E_{j}, s, 0\right) \geqq \delta_{j}>0, j=1,2$, for some $s>0$. For the estimates of this section it is important to find a lower bound in terms of $\delta_{1}, \delta_{2}$, and $n$ for the quantity

$$
M\left(\Delta\left(E_{1}, E_{2} ; A\right)\right)
$$

where $A$ is the spherical ring $R(\lambda s, s / \lambda)$ and $\lambda>1$ is an appropriately chosen number depending only on $\delta_{1}, \delta_{2}$, and $n$. Applying the comparison principle of Lemma 2.9 with $F_{1}=E_{1} \cap \bar{B}^{n}(s), F_{2}=E_{2} \cap \bar{B}^{n}(s)$, and $F_{3}=S^{n-1}(2 s)$, we get the lower bound

$$
M\left(\Delta\left(E_{1}, E_{2} ; R^{n}\right)\right) \geqq 3^{-n} \min \left\{\delta_{1}, \delta_{2}, c_{n} \log 2\right\} .
$$

Utilizing this lower bound and the upper bound of (2.4) we shall now give a number $\lambda>1$ with the desired property.

3.1. Lemma. Let $\delta_{1}, \delta_{2}>0$ and let $\lambda>1$ be such that

$$
\omega_{n-1}(\log \sqrt{\lambda})^{1-n} \leqq t / 6
$$

where $t=3^{-n} \min \left\{\delta_{1}, \delta_{2}, c_{n} \log 2\right\}$. If $s>0$ and $E_{1}$ and $E_{2}$ are two sets in $R^{n}$ with $M\left(E_{j}, s, 0\right) \geqq \delta_{j}$ for $j=1,2$, the following lower bound holds:

$$
M\left(\Delta\left(E_{1}, E_{2} ; R(\lambda s, s / \lambda)\right)\right) \geqq t / 2 .
$$

Proof. Denote by $F_{1}, F_{2}$, and $F_{3}$ the sets $\bar{R}(s, s / \sqrt{\lambda}) \cap E_{1}, \bar{R}(s, s / \sqrt{\lambda}) \cap E_{2}$, and $S^{n-1}(2 s)$, respectively. From the choice of $\lambda$ and (2.4) it follows that

$$
M\left(\bar{B}^{n}(s / \sqrt{\lambda}), s, 0\right) \leqq t / 6 .
$$

This implies for $j=1,2$ that

$$
M\left(F_{j}, s, 0\right) \geqq \delta_{j}-\frac{t}{6} \geqq 3^{n} \frac{5 t}{6} .
$$

Hence by the comparison principle of Lemma 2.9,

$$
M\left(\Delta\left(F_{1}, F_{2} ; R^{n}\right)\right) \geqq 3^{-n} \min \left\{3^{n} \frac{5 t}{6}, c_{n} \log 2\right\} \geqq \frac{5 t}{6} .
$$


Since $F_{j} \subset \bar{R}(s, s / \sqrt{\lambda}), j=1,2$, we get by (2.4) and the choice of $\lambda$,

$$
\begin{aligned}
M\left(\Delta\left(F_{1}, F_{2} ; R(\sqrt{\lambda} s, s / \lambda)\right)\right) & \geqq M\left(\Delta\left(F_{1}, F_{2} ; R^{n}\right)\right)-2 \omega_{n-1}(\log \sqrt{\lambda})^{1-n} \\
& \geqq \frac{5 t}{6}-\frac{2 t}{6}=\frac{t}{2},
\end{aligned}
$$

which together with the estimate

$$
M\left(\Delta\left(E_{1}, E_{2} ; R(\lambda s, s / \lambda)\right)\right) \geqq M\left(\Delta\left(F_{1}, F_{2} ; R(\sqrt{\lambda} s, s / \lambda)\right)\right)
$$

yields the desired lower bound.

We now prove the estimate (1.1) in the introduction.

3.2. Theorem. Let $\delta_{1}, \delta_{2}>0$ and let $\lambda>1$ be the number in Lemma 3.1. Then there exists a constant $c>0$ depending only on $\delta_{1}, \delta_{2}$, and $n$ with the following property: if $r \in\left(0, \lambda^{-1}\right]$ and $E_{1}, E_{2} \subset R^{n}$ with $M\left(E_{j}, s, 0\right) \geqq \delta_{j}$ for $s \in[\lambda r, 1]$ and $j=1,2$, then

$$
M\left(\Gamma_{r}\right) \geqq c \log \frac{1}{r}
$$

where $\Gamma_{r}=\Delta\left(E_{1}, E_{2} ; R^{n} \backslash \bar{B}^{n}(r)\right)$.

Proof. Fix $r \in\left(0, \lambda^{-1}\right]$. Define $m=\max \left\{k \in N: \lambda^{1-2 k} \geqq r\right\}$. Then $\lambda^{-3 m} \leqq r \leqq$ $\lambda^{1-2 m}$ and $m$ is a positive integer with $m \geqq(\log (1 / r) /(3 \log \lambda))$. The path families

$$
\Gamma_{k}=\Delta\left(E_{1}, E_{2} ; R\left(\lambda^{3-2 k}, \lambda^{1-2 k}\right)\right), \quad k=1, \ldots, m,
$$

are separate in the sense of $[10,6.7]$ and $\bigcup_{k=1}^{m} \Gamma_{k} \subset \Gamma_{r}$. Hence

$$
M\left(\Gamma_{r}\right) \geqq M\left(\bigcup_{k=1}^{m} \Gamma_{k}\right) \geqq \sum_{k=1}^{m} M\left(\Gamma_{k}\right) .
$$

From Lemma 3.1 it follows that there exists $t>0$ depending only on $\delta_{1}, \delta_{2}$, and $n$ such that $M\left(\Gamma_{k}\right) \geqq t / 2$ for each $k$. Thus we get

$$
M\left(\Gamma_{r}\right) \geqq \frac{t}{6 \log \lambda} \log \frac{1}{r}
$$

Since $\lambda$ depends only on $\delta_{1}, \delta_{2}$, and $n$, this estimate is of the desired type.

3.3. Corollary. Let $E_{j} \subset R^{n}$ with $M\left(E_{j}, s, 0\right) \geqq \delta_{j}>0$ for $s \in(0,1]$ and $j=1,2$, and let $\lambda>1$ be as in Lemma 3.1. Then for $r \in\left(0, \lambda^{-1}\right]$

$$
M\left(\Gamma_{r}\right) \geqq c \log \frac{1}{r},
$$

where $\Gamma_{r}=\Delta\left(E_{1}, E_{2} ; R^{n} \backslash \bar{B}^{n}(r)\right)$ and $c$ is as in Theorem 3.2. 
3.4. Theorem. Let $\delta>0$ and let $\lambda>1$ be the number in Lemma 3.1 corresponding to the case $\delta_{1}=\delta_{2}=\delta$. Then there is a number $d>0$ depending only on $\delta$ and $n$ with the following property: if $r \in\left(0, \lambda^{-2}\right]$ and $E \subset R^{n}$ with $M(E, s, 0) \geqq \delta$ for $s \in[\lambda r, 1]$, and $F_{r}$ is a continuum joining $S^{n-1}(r)$ and $S^{n-1}$, then

$$
M\left(\Gamma_{r}\right) \geqq d \log \frac{1}{r},
$$

where $\Gamma_{r}=\Delta\left(E, F_{r} ; R^{n} \backslash \bar{B}^{n}(r)\right)$.

Proof. Fix $r \in\left(0, \lambda^{-2}\right]$. Since by Lemma $2.8 M\left(F_{r}, s, 0\right) \geqq c_{n} \log \left(2-\lambda^{-1}\right)$ for $s \in[\lambda r, 1]$ it follows from Theorem 3.2 that

$$
M\left(\Delta\left(E, F_{r} ; R^{n} \backslash \bar{B}^{n}(s)\right)\right) \geqq c \log \frac{1}{s}
$$

for $s \in[\lambda r, 1]$, where $c$ is the positive constant given by Theorem 3.2 for $\delta_{1}=\delta$ and $\delta_{2}=c_{n} \log \left(2-\lambda^{-1}\right)$. Then

$$
M\left(\Gamma_{r}\right) \geqq M\left(\Delta\left(E, F_{r} ; R^{n} \backslash \bar{B}^{n}(s)\right)\right)
$$

for $s \in[\lambda r, 1]$ and hence

$$
M\left(\Gamma_{r}\right) \geqq c \log \frac{1}{\lambda r} \geqq \frac{c}{2} \log \frac{1}{r},
$$

where in the last step we have used the fact $r \in\left(0, \lambda^{-2}\right]$. We have proved the asserted estimate with $d=c / 2$.

In the next result we show that one may remove the restriction $r \in\left(0, \lambda^{-2}\right]$ of Theorem 3.4 if one slightly modifies $\Gamma_{r}$ and $d$.

3.5. Theorem. Let $\delta>0$ and let $E$ be a set in $R^{n}$ with $M(E, s, 0) \geqq \delta$ for $s \in(0,1]$. Then there is a number $d^{*}>0$ depending only on $\delta$ and $n$ such that if $r \in(0,1)$ and $F_{r}$ is a continuum joining $S^{n-1}(r)$ and $S^{n-1}$, then

where $\Gamma_{r}^{*}=\Delta\left(E, F_{r} ; R^{n}\right)$.

$$
M\left(\Gamma_{r}^{*}\right) \geqq d^{*} \log \frac{1}{r}
$$

Proof. Let $\lambda>1$ be the number in Lemma 3.1 corresponding to the case $\delta_{1}=\delta_{2}=\delta$. If $r \in\left(0, \lambda^{-2}\right]$, the desired estimate follows from Theorem 3.4 with $d^{*}=d$. Fix $r \in\left(\lambda^{-2}, 1\right)$. Applying the comparison principle of Lemma 2.9 to the sets $\bar{B}^{n} \cap E$, $\bar{B}^{n} \cap F_{r}$, and $S^{n-1}(2)$ we get

$$
M\left(\Gamma_{r}^{*}\right) \geqq 3^{-n} \min \left\{\delta, M\left(F_{r}, 1,0\right), c_{n} \log 2\right\} .
$$

In combination with the lower bound of Lemma 2.8 (2) this estimate yields

$$
M\left(\Gamma_{r}^{*}\right) \geqq 3^{-n} \min \left\{\delta, c_{n} \log (2-r)\right\} .
$$


Let $a=\left(3^{n} \log \lambda^{2}\right)^{-1} \min \left\{\delta, c_{n} \log \left(2-\lambda^{-2}\right)\right\}$. Since $r \in\left(\lambda^{-2}, 1\right)$ we obtain

$$
M\left(\Gamma_{r}^{*}\right) \geqq a \log \frac{1}{r} .
$$

Hence

$$
M\left(\Gamma_{r}^{*}\right) \geqq d^{*} \log \frac{1}{r}
$$

for all $r \in(0,1)$, where $d^{*}=\min \{d, a\}>0$.

3.6. Remark. In Lemma 3.1 we assumed that $M\left(E_{j}, s, 0\right) \geqq \delta_{j}>0$ for $j=1,2$ and obtained a lower bound for $M(\Gamma(\lambda s, s / \lambda))$, where $\Gamma(\lambda s, s / \lambda)=\Delta\left(E_{1}, E_{2} ; R(\lambda s, s / \lambda)\right)$. If we assume that $M\left(E_{j} \cap B^{n}(s), s, 0\right) \geqq \delta_{j}>0$ for $j=1,2$, we can prove a related lower bound for $M(\Gamma(s, s / \sqrt{\lambda}))$ by making use of (2.4) and the reflection principle for the modulus (cf. Lemma 4.5).

3.7. Remark. Observe that the lower bound of Theorem 3.2 follows from the cap-inequality, Lemma 2.7, in certain special cases, e.g. when both $E_{1}$ and $E_{2}$ meet $S^{n-1}(r)$ for each $r \in(0,1]$ (cf. [10, 10.14]). However, the condition of Theorem 3.2 may be satisfied even if $\left(E_{1} \cup E_{2}\right) \cap S^{n-1}(r)=\emptyset$ for almost every $r \in(0,1]$. In fact, by a result of Wallin there are sets $E_{1}, E_{2}$ with $M\left(E_{j}, r, 0\right) \geqq \delta_{j}>0$ for every $r \in(0,1] j=1,2$, such that the Hausdorff dimension of $E_{j}$ is zero, $j=1,2$ (see $[13,2.5(3)])$. Various sufficient conditions for cap dens $(E, 0)>0$ were given in [13, Section 2] and in Martio [5].

Let $E_{1}$ and $E_{2}$ be two sets with cap dens $\left(E_{j}, 0\right)>0, j=1,2$, and for $r>0$ let $\Gamma_{r}=\Delta\left(E_{1}, E_{2} ; R^{n} \backslash \bar{B}^{n}(r)\right)$. Then Theorem 3.2 shows that $M\left(\Gamma_{r}\right)$ tends to infinity with a certain rapidity when $r \rightarrow 0$. In the next two theorems we study the behavior of $M\left(\Gamma_{r}\right)$ under the more general assumptions that cap dens $\left(E_{1}, 0\right)>0$, cap $\overline{\operatorname{dens}}\left(E_{2}, 0\right)>0$. We show that

$$
\lim _{r \rightarrow 0} M\left(\Gamma_{r}\right)=\infty
$$

also in this case, but the convergence may take place as slowly as we wish.

3.8. Theorem. Let $E_{1}$ and $E_{2}$ be two sets with cap dens $\left(E_{1}, 0\right)=\delta_{1}>0$ and cap $\overline{\operatorname{dens}}\left(E_{2}, 0\right)=\delta_{2}>0$, and let $\Gamma_{r}=\Delta\left(E_{1}, E_{2} ; R^{n} \backslash \bar{B}^{n} \overline{(r))}\right.$ for $\quad r>0$. Then $M\left(\Gamma_{r}\right) \rightarrow \infty$ as $r \rightarrow 0$.

Proof. Choose a sequence $\left(r_{k}\right)$ tending to zero such that $M\left(E_{j}, r_{k}, 0\right) \geqq \delta_{j} / 2$, $j=1,2$, for every $k=1,2, \ldots$. Let $\lambda>1$ and $t>0$ be the constants corresponding to $\delta_{1} / 2, \delta_{2} / 2$, and $n$ given by Lemma 3.1. Passing to a subsequence and relabeling if necessary, we may assume that the rings $R\left(\lambda r_{k}, r_{k} / \lambda\right), k=1,2, \ldots$ are separate. Let $\Gamma_{k}=\Delta\left(E_{1}, E_{2} ; R\left(\lambda r_{k}, r_{k} / \lambda\right)\right), k=1,2, \ldots$. Since the families $\Gamma_{k}$ are separate and $M\left(\Gamma_{k}\right) \geqq t / 2>0$ for all $k$, the assertion follows from [10, 6.7].

3.9. Theorem. Let $h:(0,1] \rightarrow(0, \infty)$ be a non-increasing function with $\lim _{t \rightarrow 0+} h(t)=\infty$. Then there exist sets $E$ and $F$ with cap dens $(E, 0)>0$ and cap $\overline{\operatorname{dens}}(F, 0)>0 \quad$ such that $M\left(\Gamma_{r}\right) \leqq h(r)$ for all $r \in(0,1]$, where $\Gamma_{r}=$ $\Delta\left(E, F ; R^{n} \backslash \bar{B}^{n}(r)\right)$. 
Proof. Let $E_{k}=S^{n-1}\left(2^{-2 k}\right), F_{k}=S^{n-1}\left(2^{-2 k+1}\right), k=1,2, \ldots, \quad$ and $E=\cup E_{k}$. Then cap dens $(E, 0) \geqq c_{n} \log (5 / 3)$ by Lemma 2.8 (1). We shall now choose an infinite set $P \subset N$ such that the set $F=\cup\left\{F_{k}: k \in P\right\}$ has the desired property. Observe that for any infinite set $P \subset N$ cap $\overline{\operatorname{dens}}(F, 0) \geqq c_{n} \log 3$ by Lemma 2.8 (1). If $k \geqq 2$, then by [10, 7.5, 6.2, 6.4]

$$
M\left(\Delta\left(F_{k}, E ; R^{n}\right)\right)=2 \omega_{n-1}(\log 2)^{1-n}=b .
$$

For $k \geqq 1$ and $0<r<2^{-2 k+1}$

Let

$$
M\left(\Delta\left(F_{k}, E ; R^{n} \backslash \bar{B}^{n}(r)\right)\right) \leqq b .
$$

$$
\begin{gathered}
p_{1}=\min \left\{k \in N: h\left(2^{-2 k+2}\right) \geqq b\right\} \\
p_{m+1}=\min \left\{k \in N, k>p_{m}: h\left(2^{-2 k+2}\right) \geqq(m+1) b\right\} \\
m=1,2, \ldots .
\end{gathered}
$$

We show that the set $P=\left\{p_{k}: k \in N\right\}$ has the asserted property. Fix $r \in(0,1]$. If $r \geqq 2^{-2 p_{1}+1}$, there is nothing to prove, since then $M\left(\Gamma_{r}\right)=M(\emptyset)=0<h(r)$. Hence we may assume $r \in\left(0,2^{-2 p_{1}+1}\right)$. Let

Then by $[10,6.2]$

$$
s=\max \left\{k \in N: 2^{-2 p_{k}+1} \geqq r\right\} \geqq 1 .
$$

as desired.

$$
M\left(\Gamma_{\mathrm{r}}\right) \leqq s b \leqq h\left(2^{-2 p_{s}+2}\right) \leqq h(r)
$$

3.10. Remark. In Theorem 3.8 one may not replace the assumptions by cap $\overline{\operatorname{dens}}\left(E_{j}, 0\right)>0, j=1,2$. To show this we construct for a given $\varepsilon>0$ sets $E_{1}$ and $E_{2}$ with $M\left(\Delta\left(E_{1}, E_{2} ; R^{n}\right)\right)<\varepsilon$ and cap $\overline{\operatorname{dens}}\left(E_{j}, 0\right)>0, j=1,2$.

Let $\varepsilon>0$ and $r_{1}=1$. Choose $r_{k+1} \in\left(0, r_{k} / 2\right), k=1,2, \ldots$ such that

$$
\omega_{n-1}\left(\log \frac{r_{k}}{r_{k+1}}\right)^{1-n}<\varepsilon 2^{-k} \text {. }
$$

Then it follows from (2.4) that the sets $E_{1}=\bigcup_{k=0}^{\infty} S^{n-1}\left(r_{2 k+1}\right)$ and $E_{2}=\bigcup_{k=1}^{\infty} S^{n-1}\left(r_{2 k}\right)$ satisfy $M\left(\Delta\left(E_{1}, E_{2} ; R^{n}\right)\right)<\varepsilon$. From Lemma 2.8 (1) it follows that cap $\overline{\operatorname{dens}}\left(E_{j}, 0\right)>0$, $j=1,2$.

\section{Non-tangential absolute values of quasiconformal maps}

In the present section we shall use the method of Section 3 to study boundary behavior of quasiconformal mappings. A homeomorphism $f: G \rightarrow G^{\prime}$, where $G$ and $G^{\prime}$ are domains in $R^{n}$, is quasiconformal if there exists a constant $K \in[1, \infty)$ such that for every path family $\Gamma$ in $G$

$$
M(\Gamma) / K \leqq M(f \Gamma) \leqq K M(\Gamma),
$$

where $f \Gamma=\{f \circ \gamma: \gamma \in \Gamma\}$. The smallest possible $K$ is denoted by $K(f)$. 
Let $f$ be a quasiconformal mapping of $B^{n}$, let $b \in \partial B^{n}$, and let $E \subset B^{n}$ be a set with cap dens $(E, b)>0$. The first theorem of this section shows that each nontangential lim sup of the absolute value of $f$ is bounded by the lim sup of the absolute value of $f$ through the set $E$. As a consequence we get an extension of Tord Hall's theorem [4, Theorem II], which was proved in [13, 4.4] by different methods. The second and the last theorem of this section gives an alternative proof for the quasiconformal counterpart of J. L. Doob's theorem in $[13,5.5]$.

4.2. The hyperbolic metric. The hyperbolic metric $\varrho$ in $B^{n}$ is defined by the element of length

$$
d \varrho=\frac{|d x|}{1-|x|^{2}}
$$

If $a$ and $b$ are points of $B^{n}$, then $\varrho(a, b)$ denotes the geodesic distance between $a$ and $b$ corresponding to this element of length. For $b \in B^{n}$ and $M \in(0, \infty)$ we let $D(b, M)$ denote the hyperbolic ball $\left\{x \in B^{n}: \varrho(b, x)<M\right\}$. Let $r_{b}=\min \{|z-b|: z \in \partial D(b, M)\}$. By integrating we get

$$
r_{b}=\frac{\left(1-|b|^{2}\right) \tanh M}{1+|b| \tanh M} .
$$

The next result follows from the proof of $[13,6.5]$.

4.4. Lemma. Let $f: B^{n} \rightarrow G^{\prime}$ be a quasiconformal mapping and let $\left(b_{k}\right)$ be a sequence in $B^{n}$ with $\left|b_{k}\right| \rightarrow 1$ as $k \rightarrow \infty$. If $M \in(0, \infty)$ and $E=\cup D\left(b_{k}, M\right)$, then

$$
\limsup _{\substack{|x| \rightarrow 1 \\ x \in E}}|f(x)|=\limsup _{k \rightarrow \infty}\left|f\left(b_{k}\right)\right| \text {. }
$$

A corresponding result holds for $\lim$ inf.

We shall need the following symmetry property for the modulus, which was proved in [13, Section 4].

4.5. Lemma. Let $E$ and $F$ be two subsets of $B^{n}$. Then $M\left(\Delta\left(E, F ; B^{n}\right)\right) \geqq$ $\left.M\left(E, F ; R^{n}\right)\right) / 2$.

For $b \in \partial B^{n}$ and $\varphi \in(0, \pi / 2)$ we let $K(b, \varphi)$ denote the cone $\left\{z \in R^{n}:(b \mid b-z)>\right.$ $|b-z| \cos \varphi\}$.

4.6. Theorem. Let $f: B^{n} \rightarrow G^{\prime}$ be a quasiconformal mapping, let $b \in \partial B^{n}$, and let $E \subset B^{n}$ be a set with cap dens $(E, b)>0$. Then for every $\varphi \in(0, \pi / 2)$

$$
\begin{aligned}
& \limsup _{\substack{x \rightarrow b \\
x \in K(b, \varphi)}}|f(x)| \leqq \limsup _{\substack{x \rightarrow b \\
x \in E}}|f(x)|, \\
& \liminf _{\substack{x \rightarrow b \\
x \in E}}|f(x)| \leqq \liminf _{\substack{x \rightarrow b \\
x \in K(b, \varphi)}}|f(x)| .
\end{aligned}
$$


Proof. Fix $\varphi \in(0, \pi / 2)$. It suffices to prove the first inequality, since the second one can be proved in the same way. Denote by $\tilde{s}$ and $\tilde{t}$ the left and right hand sides of the first inequality, respectively. Assume that $\tilde{s}>\tilde{t}$. Choose $t, s \in(\tilde{t}, \tilde{s})$ with $t<s$. By Lemma 4.4 there is a sequence $\left(a_{k}\right)$ in $B^{n} \cap K(b, \varphi)$ with $a_{k} \rightarrow b$ as $k \rightarrow \infty$ and with $|f(x)|>s$ for all $x \in \cup D\left(a_{k}, 1\right)=F$. Choose $r_{1} \in(0,1)$ such that $|f(x)|<t$ for $x \in E_{1}=E \cap B^{n}\left(b, r_{1}\right)$. Since $a_{k} \in K(b, \varphi)$ and $a_{k} \rightarrow b$, there exists an integer $k_{0}$ such that for $k \geqq k_{0}$

$$
\frac{1-\left|a_{k}\right|}{\left|a_{k}-b\right|} \geqq(\cos \varphi) / 2>0 .
$$

Write $r_{k}=\min \left\{\left|z-a_{k}\right|: z \in \partial D\left(a_{k}, 1\right)\right\}$. For $k \geqq k_{0}$ we obtain by (4.3)

$$
\frac{r_{k}}{\left|a_{k}-b\right|} \geqq \frac{r_{k}}{1-\left|a_{k}\right|}(\cos \varphi) / 2 \geqq(\tanh 1 \cos \varphi) / 2 .
$$

By Lemma 2.8 (2) this implies that cap $\overline{\operatorname{dens}}(F, b)>0$. Let $\Gamma=\Delta\left(E_{1}, F ; B^{n}\right)$. It follows from Lemma 4.5 and Theorem 3.8 that $M(\Gamma)=\infty$. This conclusion contradicts (4.1) and the upper bound

given by (2.4).

$$
M(f \Gamma) \leqq \omega_{n-1}\left(\log \frac{s}{t}\right)^{1-n}
$$

4.7. Corollary. Let $f: B^{n} \rightarrow G^{\prime}$ be a quasiconformal mapping, let $b \in \partial B^{n}$, and let $E, F \subset B^{n}$ be two sets with cap dens $(E, b)>0$ and cap $\overline{\operatorname{dens}}(F, b)>0$. Suppose that $f(x)$ tends to a limit $\alpha$ as $x$ approaches $b$ through the set $F$. Then $|\alpha| \leqq$ $\lim \sup _{x \rightarrow b, x \in E}|f(x)|$.

Proof. The proof follows from the proof of Theorem 4.6.

4.8. Remark. It is not possible to replace the condition cap dens $(E, b)>0$ of Corollary 4.7 by cap $\overline{\operatorname{dens}}(E, b)>0$. We shall now show this with the aid of the following argument, which resembles the reasoning in $[13,6.6]$.

Let $f: B^{2} \rightarrow G^{\prime}$ be a conformal mapping which does not possess a radial limit at $e_{1}=(1,0) \in \partial B^{2}$. We may assume that $0, \alpha \in C_{\mathrm{rad}}\left(f, e_{1}\right)$, where $\alpha \neq 0$ and $C_{\mathrm{rad}}\left(f, e_{1}\right)$ is the cluster set of $f$ on the radius $\left(0, e_{1}\right)$. Choose sequences $\left(a_{k}\right)$ and $\left(b_{k}\right)$ in $\left(0, e_{1}\right)$ with $a_{k} \rightarrow e_{1}$ and $b_{k} \rightarrow e_{1}$ such that $f\left(a_{k}\right) \rightarrow 0$ and $f\left(b_{k}\right) \rightarrow \alpha$ as $k \rightarrow \infty$. Write $E=\cup D\left(a_{k}, 1\right)$ and $F=\cup D\left(b_{k}, 1\right)$. From Lemma 4.4 it follows that $f(x) \rightarrow 0$ as $x \rightarrow e_{1}$ through the set $E$ and $f(x) \rightarrow \alpha$ as $x \rightarrow e_{1}$ through $F$. Lemma 2.8 (2) implies that cap $\overline{\operatorname{dens}}\left(E, e_{1}\right)>0$ and cap $\overline{\operatorname{dens}}\left(F, e_{1}\right)>0$. Hence the assumption cap dens $(E, b)>0$ of Corollary 4.7 cannot be replaced by cap $\overline{\operatorname{dens}}(E, b)>0$.

We now give a consequence of Theorem 4.6, which was proved in [13] by different methods. This consequence extends Tord Hall's theorem [4, Theorem II] on bounded analytic functions (see [13, Section 4]). See also F. W. Gehring's result in $[2$, p. 21]. 
4.9. Corollary. Let $f: B^{n} \rightarrow G^{\prime}$ be a quasiconformal mapping and let $f(x)$ tend to a limit $\alpha$ as $x$ approaches $b \in \partial B^{n}$ through a set $E$ in $B^{n}$ with cap dens $(E, b)>0$. Then $f$ has the angular limit $\alpha$ at $b$.

4.10. Cluster values. Given a continuous mapping $f: B^{n} \rightarrow R^{n}, \varepsilon>0$, and $\alpha \in \bar{R}^{n}$, we denote by $E_{\varepsilon}$ the set $f^{-1} B^{n}(\alpha, \varepsilon)$ when $\alpha \neq \infty$ and $f^{-1}\left(R^{n} \backslash \bar{B}^{n}(1 / \varepsilon)\right)$ when $\alpha=\infty$. Then the cluster set $C(f, b)$ of $f$ at $b$ (cf. [10, p. 52]) can be alternatively defined as the set of all points $\alpha \in \bar{R}^{n}$ such that $b \in \bar{E}_{\varepsilon}$ for all $\varepsilon>0$.

Let now $f: B^{n} \rightarrow G^{\prime}$ be quasiconformal and $b \in \partial B^{n}$. Then Corollary 4.9 gives us a sufficient condition for the fact that a point $\alpha$ is the angular limit of $f$ at $b$. The next theorem provides us with a more general result of this kind, and for this purpose we introduce some terminology (cf. [13, Section 5]). Let $\alpha \in C(f, b)$ and for $\varepsilon>0$ write $\delta_{\varepsilon}=$ cap dens $\left(E_{\varepsilon}, b\right)$. Then $\alpha$ is a capacity cluster value of $f$ at $b$ if for some $d>0$

$$
\lim _{\varepsilon \rightarrow 0} \varepsilon_{\varepsilon}^{\delta_{\varepsilon}^{d}}=0
$$

The least upper bound of numbers $d$ for which condition (4.11) holds, is called the order of $\alpha$. Adopting this terminology we shall now prove the following theorem, which extends Doob's theorem [1, Theorem 4] to the case of quasiconformal mappings. Theorem 4.12 was proved in $[13,5.5]$ by a different method involving a normal family argument. For a comparison between Doob's original theorem and 4.12, see [13, Section 5].

4.12. Theorem. Let $f: B^{n} \rightarrow G^{\prime}$ be a quasiconformal mapping, let $b \in \partial B^{n}$, and let $f$ have a capacity cluster value $\alpha$ of order greater than $1 /(n-1)$ at $b$. Then $f$ has the angular limit $\alpha$ at $b$.

Proof. Performing a preliminary Möbius transformation if necessary, we may assume that $\alpha \neq \infty$. Suppose that $f$ does not have the angular limit $\alpha$ at $b$. Then there is $\varphi \in(0, \pi / 2)$ and a sequence $\left(b_{k}\right)$ in $K(b, \varphi) \cap B^{n}$ with $b_{k} \rightarrow b$ and $f\left(b_{k}\right) \rightarrow$ $\beta \neq \alpha$ as $k \rightarrow \infty$. Fix $r_{0}>0$ such that $\beta \in \bar{R}^{n} \backslash B^{n}\left(\alpha, 2 r_{0}\right)$. For $\varepsilon \in\left(0, r_{0}\right)$ let $E_{\varepsilon}=$ $f^{-1} B^{n}(\alpha, \varepsilon)$. Since $b_{k} \in K(b, \varphi)$ and $b_{k} \rightarrow b$, there is $k_{1}$ such that $1-\left|b_{k}\right| \geqq$ $\left|b_{k}-b\right|(\cos \varphi) / 2$ for $k \geqq k_{1}$. By Lemma 4.4 there is $k_{0} \geqq k_{1}$ such that $f D\left(b_{k}, 1\right) \subset$ $R^{n} \backslash B^{n}\left(\alpha, r_{0}\right)$ for $k \geqq k_{0}$. Let $E=\bigcup_{k \geqq k_{0}} D\left(b_{k}, 1\right)$. By $(4.3) B^{n}\left(b_{k}\right.$, $\left.(\tanh 1)\left(1-\left|b_{k}\right|\right)\right) \subset$ $D\left(b_{k}, 1\right)$ for all $k=1,2, \ldots$. Hence it follow's from Lemma 2.8 (2) that for $k \geqq k_{0}$

$$
M\left(E,\left|b_{k}-b\right|, b\right) \geqq c(n, \varphi)=c_{n} \log (1+(\tanh 1 \cos \varphi) / 2) .
$$

For $\varepsilon \in\left(0, r_{0}\right)$ write $\Gamma_{\varepsilon}=\Delta\left(E, E_{\varepsilon} ; B^{n}\right)$. Let $\delta_{\varepsilon}=$ cap dens $\left(E_{\varepsilon}, b\right)$. Then for $\varepsilon \in\left(0, r_{0}\right)$ there is $k_{\varepsilon} \geqq k_{0}$ such that $M\left(E_{\varepsilon},\left|b_{k_{\varepsilon}}-b\right|, b\right) \geqq \delta_{\varepsilon} / 2$. For $\varepsilon \in\left(0, r_{0}\right)$ let $F_{1}^{\varepsilon}=E \cap$ $\bar{B}^{n}\left(b,\left|b_{k_{\varepsilon}}-b\right|\right), \quad F_{2}^{\varepsilon}=E_{\varepsilon} \cap \bar{B}^{n}\left(b,\left|b_{k_{\varepsilon}}-b\right|\right), \quad$ and $\quad F_{3}^{\varepsilon}=S^{n-1}\left(b, 2\left|b_{k_{\varepsilon}}-b\right|\right)$. Because $\Delta\left(F_{1}^{\varepsilon}, F_{2}^{\varepsilon} ; B^{n}\right) \subset \Gamma_{\varepsilon}$ we get by the comparison principle of Lemma 2.9 and by Lemma 4.5

$$
M\left(\Gamma_{\varepsilon}\right) \geqq 2^{-1} \cdot 3^{-n} \min \left\{\delta_{\varepsilon} / 2, c(n, \varphi), c_{n} \log 2\right\}
$$


for $\varepsilon \in\left(0, r_{0}\right)$. By (2.4) we get

$$
M\left(f \Gamma_{\varepsilon}\right) \leqq \omega_{n-1}\left(\log \frac{r_{0}}{\varepsilon}\right)^{1-n}
$$

for $\varepsilon \in\left(0, r_{0}\right)$. This together with the preceding lower bound for $M\left(\Gamma_{\varepsilon}\right)$ and (4.1) shows that $\delta_{\varepsilon} \rightarrow 0$ as $\varepsilon \rightarrow 0$. Hence there exists $r_{1} \in\left(0, r_{0}\right)$ such that $M\left(\Gamma_{\varepsilon}\right) \geqq 3^{-n-2} \delta_{\varepsilon}$ for $\varepsilon \in\left(0, r_{1}\right)$. This lower bound, together with the above upper bound for $M\left(f \Gamma_{\varepsilon}\right)$ and (4.1), yields for $\varepsilon \in\left(0, r_{1}\right)$

$$
0<\left(3^{n+2} K(f) \omega_{n-1}\right)^{-1} \leqq\left(\log r_{0}^{\beta_{\varepsilon}}-\log \varepsilon^{\beta_{\varepsilon}}\right)^{1-n},
$$

where $\beta_{\varepsilon}=\delta_{\varepsilon}^{1 /(n-1)}$. Since $\alpha$ is a capacity cluster value of order greater than $1 /(n-1)$, condition (4.11) is satisfied with $d=1 /(n-1)$ and thus (4.13) yields a contradiction when $\varepsilon$ tends to zero.

If we examine the proof of Theorem 4.12 we see that the following result holds.

4.14. Corollary. Let $f: B^{n} \rightarrow G^{\prime}$ be a quasiconformal mapping, let $b \in \partial B^{n}$, and let $E_{\varepsilon}=f^{-1} B^{n}(\varepsilon), \delta_{\varepsilon}=$ cap dens $\left(E_{\varepsilon}, b\right)$. If $\lim \sup _{\varepsilon \rightarrow 0} \delta_{\varepsilon}(\log (1 / \varepsilon))^{n-1}=\infty$, then $f$ has angular limit 0 at $b$.

4.15. Remarks. (1) The assumption of Theorem 4.12 implies that

$$
\lim _{\varepsilon \rightarrow 0} \delta_{\varepsilon}(\log (1 / \varepsilon))^{n-1}=\infty .
$$

Hence the assumption of Corollary 4.14 is slightly more general.

(2) For further results connected with Corollary 4.9 and Theorem 4.12 we refer the reader to [13]. Observe that these results can be easily generalized to cover the case of closed quasiregular mappings as well (cf. [12]). For the theory of general quasiregular mappings we refer the reader to the papers of Martio, Rickman, and Väisälä (cf. [6] and the references in [11]) and for the theory of closed quasiregular mappings to [11, Chapter II].

(3) It is possible to extend Corollary 4.9 to the case when the set $E$ is a compact set on the boundary of $B^{n}$. Perhaps the most natural way to do this is to introduce the asymptotic extension $f$ of a quasiconformal mapping $f$ of $B^{n}$ (cf. Näkki [8]) and then to define the values of $f$ on $E$ in terms of $\dot{f}$. Since $E$ is compact, we can use a result of Gehring [3, Lemma 1] in place of Lemma 4.5. We can also extend Theorem 4.12 in the same way.

Acknowledgements. This research was done during the academic year 1977-78, when the author was visiting the Mittag-Leffler Institute. The author is grateful to the Royal Swedish Academy of Sciences for its financial support. 


\title{
References
}

[1] Doob, J. L.: The boundary values of analytic functions. - Trans. Amer. Math. Soc. 34, 1932, 153-170.

[2] Gehring, F. W.: The Carathéodory convergence theorem for quasiconformal mappings in space. - Ann. Acad. Sci. Fenn. Ser. A I 336/11, 1963, 1-21.

[3] Gehring, F. W.: A remark on domains quasiconformally equivalent to a ball. - Ibid. 2, 1976, 147-155.

[4] Hall, T.: Sur la mesure harmonique de certains ensembles. - Ark. Mat. Astr. Fys. 25 A, No. 28, 1937, 1-8.

[5] Martio, O.: Capacity and measure densities. - Ann. Acad. Sci. Fenn. Ser. A I 4, 1978/1979, $109-118$.

[6] Martio, O., S. Rickman, and J. VÄISÄLÄ: Distortion and singularities of quasiregular mappings. - Ibid. 465, 1970, 1-13.

[7] Martio, O., and J. Sarvas: Density conditions in the $n$-capacity. - Indiana Univ. Math. J. 26, 1977, 761-776.

[8] NäKKI, R.: Extension of Loewner's capacity theorem. - Trans. Amer. Math. Soc. 180, 1973, 229-236.

[9] NÄKKI, R.: Prime ends and quasiconformal mappings. - J. Analyse Math. (to appear).

[10] V̈̈̈SÄL $\ddot{A}$, J.: Lectures on $n$-dimensional quasiconformal mappings. - Lecture Notes in Mathematics 229, Springer-Verlag, Berlin-Heidelberg-New York, 1971.

[11] VUORINen, M.: Exceptional sets and boundary behavior of quasiregular mappings in $n$-space. Ann. Acad. Sci. Fenn. Ser. A I Math. Dissertationes 11, 1976, 1-44.

[12] Vuorinen, M.: On angular limits of closed quasiregular mappings. - Proceedings of the First Finnish-Polish Summer School in Complex Analysis (Held at Podlesice, Poland, June 16-22, 1977), Part II, edited by J. Lawrynowicz and O. Lehto, University of Łódź, Łódź, 1978, pp. 69-74.

[13] Vuorinen, M.: On the existence of angular limits of $n$-dimensional quasiconformal mappings. Ark. Mat. (to appear).

[14] VUORINEN, M.: Lower bounds for the $n$-moduli of path families with applications to boundary behavior of quasiconformal and quasiregular mappings. - Proceedings of Colloquium on Complex Analysis, Joensuu, Finland, August 24-27, 1978, edited by I. Laine, O. Lehto, and T. Sorvali, Lecture Notes in Mathematics 747, Springer-Verlag, Berlin-Heidelberg-New York, 1979 (to appear).

[15] Zremer, W. P.: Extremal length and p-capacity. - Michigan Math. J. 16, 1969, 43-51.

\author{
University of Helsinki \\ Department of Mathematics \\ SF-00100 Helsinki 10
}

Finland

Received 17 November 1978

Revision received 5 February 1979 